\title{
Incidencia de la Calidad del Sistema de Formación Profesional en la Satisfacción de los Estudiantes de la Facultad de Ciencias Administrativas de la Universidad Nacional Jorge Basadre Grohmann de Tacna, período 2009
}

\author{
Impact of the Quality of Vocational Training System in the \\ Satisfaction of the Students of the Faculty of Administration \\ National University of Tacna Jorge Basadre Grohmann, 2009 Period \\ Silvia Munarriz O'phelan
}

RESUMEN:

El presente trabajo de investigación se llevó a cabo con el propósito de determinur la incidencia de la calidad del sistema de formación profesional en la satisfacciom de los estudiantes de la Facultad de Ciencias Administrativas de la UN.JBG de Tacna, periodo 200)9. Para ello se estableció la siguiente hipótesis: La calidad del Sistema de Formación Profesional incide en el Nivel de Satisfacción de los Estudiantes de la Facultad de Ciencias Administrativas de la UNJBG de Tacna, periodo 2009. El tipo de investigación es bisica, porque se realiza principalmente para conocer los fundamentos de los fenómenos sin atender a aplicactiones particulares. Es descriptiva correlacional. puesto que husca medir las variables y recoger información sobre la relación de la Calidad del Sistema de Formación Profesional y la Satisfacción de los Estudiantes de la Faculiad de Ciencias Administrativas de la UNJBG de Tacna. Los datos obtenidos se' labularon y analizaron mediante cuadros y graficos. Una vez finalizada la fase de análisis e interpretación de' los resultados se determinó que la Calidad del Sistema de Formación Profesional incide en el Nivel de Satisfacción de los Estudiantés de la Facultad de Ciencias Administrativas de la UNJBG de Tacna, periodo 2009: habiéndose encontrado que existen indicadores que no cumplen con la satisfacción de necesidades y. requerimientos de los estudiantes y futuros profesionales de las carreras de Administración e Ingenieria
Comercial.

Palabras clave: calidad, sistemade formación profesional, satisfacción.

\section{ABSTRACT:}

This research work was done in order to determine the incidence of vocational training system quality to the satisfaction of the students of the Administrative Sciences Faculty at the UN.IBG in Tacna 2009 period. For this, it was established the following hypothesis: The quatity of the profesional training system affects the level of satisfaction of students of the Administrative Sciences Faculty at the UNJBG Tucna 2009 period. The type of research is basic, becuuse it is mainly done to know the ahout fundanentals of the phenomena without regard to particular applications. It is correlational descriptive, since it is intended to me asure the variables and collect data on the relationship of the Quality of the Profesional Training System and Satisfaction of Students of the Administrative Sciences Faculty at the UN.JBG in Tacna. The data results were tabulated and analyzed using tables and graphs. Once the phase of ancilysis and interpretation of results ended, it was determined that the Quality of Vocational Training System affects the level of students satisfaction of the Administrative Sciences Faculty at the UNJBG in Tacna, period 2009. having foumd that there are indicators that do not fullfill the satisfaction of needs and requirements of students and future professionals in the careers of Business Administration and Comercial Engineering.

Keywords: quality, system training, satisfaction.

' Magister en Docencia Universitaria, Licenciada en Antropologia Social.

Facultad de Educacion. Comunicaciones y Humanidades. Universidad Nacional Jorge Basadre Crohmann 


\section{INTRODUCCIÓN}

En la actualidad, el profesional de administración de empresas tiene grandes desafios teniendo en cuenta las nuevas perspectivas futuras, representadas éstas en la globalización de la economia o internacionalización de los mercados, una competencia agresiva, crecimiento de las organizaciones y el avance tecnológico; asimismo, el vertiginoso cambio social permanente, que es hoy la constante en nuestro contexto. Razones, por la que es de suma importancia la calidad en la formación que debe tener el futuro profesional en administración, el cual debe desarrollar una serie de competencias para asi poder contrarrestar los cambios que le ofrece el entorno; entendidas éstas como el conocimiento actuado ante una situación determinada.

El problema que se expone tiene que ver con el nivel de formación académica profesional de los alumnos de Facultad de Ciencias Administrativas, los cuales no están respondiendo a las verdaderas necesidades de su campo disciplinario; teniendo en cuenta que no son los profesionales de la administración $y / 0$ a tỉnes, los que crean, direccionan o gestionan los procesos de las diferentes organizaciones de la sociedad, sino profesionales de otras disciplinas que dentro de su campo de acción no está la gestión de éstas, lo cual crea un problema desde el punto de vista laboral y de la proyección social de los programas. Los estudiantes, como es de esperarse, no están formados para crear empresa sino para ser gerentes o ejecutivos de empresas corroborándose la conjetura de que en las facultades de Administración de las Universidades Públicas se forman profesionales con "mentalidad de empleado".

Cada día, las instituciones de educación superior tienen que replantear constantemente sus planes de estudio obedeciendo a estos cambios, toda vez que por mandato legal deben de transitar por el camino hacia la excelencia académica, como to es, por ejemplo, el proceso de Acreditación Institucional, que percibe mantener universidades fortalecidas que estén en permanente cambio y que respondan a las necesidades del entorno. ya que el día en que la Universidad no responda a las necesidades del contex to ese dia pierde su razón de ser.

Preocupados por el problema de la formación y teniendo en cuenta esta problemática, con el desarrollo de la presente investigación se busca determinar la incidencia de la Calidad del Sistema de Formación Profesional en la Satisfacción de los Estudiantes de la Facultad de Ciencias Administrativas de la UNJBG de Tacna, período 2009.

\section{MATERIALESY MÉTODOS.}

\subsection{Muestreo}

\subsubsection{Ubicación de la Zona de Muestreo}

Situada en la Facultad de Ciencias Administrativas de la Universidad Nacional Jorge Basadre Grohmann de Tacna.

\subsubsection{Poblacióny Muestra}

\section{Población}

Considerando el propósito y los objetivos de la investigación, se trabajó con los estudiantes de la Facultad de Ciencias Administrativas de la UNJBG.

\section{Muesira}

Definida la población se determinó la muestra a los estudiantes de Administración; para hacer más accesible el estudio de investigación.

\section{Procedimiento para determinar la muestra}

$$
n=\frac{N Z 2}{4(n-1) e 2+Z 2}
$$

Dónde:

$\mathrm{N}=$ Población

$\mathrm{n}=$ Muestra provisional

$\mathrm{Z}=$ Nivel de confianza

$\mathrm{e}=0.05$ (precisión o margen de error)

\subsection{Téenicas e Instrumentos de Recolección de Datos}

Las técnicas utilizadas para la recolección de datos en la presente investigación fue la encuesta. aplicada a los estudiantes de la Facultad de Ciencias Administrativas de la UNJBG, con la finalidad de conocer la calidad del sistema de formación profesional de la FACA.

\subsection{Instrumentos para la Recolección de los Datos}

Mediante el cuestionario estructurado se determinará el nivel de satisfacción de los estudiantes de la Facultad de Ciencias Administrativas respecto al nivel de calidad del sistema de formación profesional de la FACA.

\subsubsection{Validacióny Confiabilidad de Instrumentos}

a) Validacion: el instrumento de medición que se aplicará será sometido al juicio de expertos a fin de evaluar el constructo del mismo y su correspondencia con las variables a estudio.

b) Confiablidad: el instrumento de medición será sometido a la prueba de confiabilidad del coeficiente Alfa de Cronbach.

\subsection{Técnicas de Análisis de datos}

La información se procesó electrónicamente, utilizando para ello el programa Microsoft Excel versión XP bajo ambiente Windows, SPSS (versión 15) para presentar los items mediante figuras $\mathrm{y}$, al mismo tiempo estimar las frecuencias absolutas y relativas de cada reactivo para describir la variable a estudio.

\section{RESULTADOS}

Con el fin de determinar la incidencia del Sistema de Formación Profesional en la Satisfacción de los 
Estudiantes de la Facultad de Ciencias Administrativas de la Universidad Nacional Jorge Basadre Grohmann de Tacna, Periodo 2009; se aplicó un cuestionario autoadministrado el cual contenía aspectos referentes al nivel de: enseñanza y aprendizaje, equipo docente , organización de las escuelas profesionales, curricula de estudios y tipo de evaluaciones; cuyos resultados son analizados a continuación en las tablas 01, 02, 03, 04 y 05 .

Entre los métodos de enseñanza y aprendizaje analizados se destacan los siguientes: clases teóricas, clases prácticas y visitas de campo y prácticas pre profesionales; cuyos resultados se destacan en la tabla 1.

\section{DISCUSIÓN DE RESULTADOS}

Los resultados mostrados en la Tabla $\mathrm{N}^{\circ} 0$ l; nos permite concluir que los estudiantes de la Escuela de Administración se encuentran insatisfechos con los métodos de enseñanza y aprendizaje, puesto que las clases proporcionadas por los docentes no satisfacen los indicadores de satisfacción señalados.

En la Tabla $\mathrm{N}^{\circ} 02$; nos permite determinar que los estudiantes se encuentran insatisfechos con la Organización de las Carreras, puesto que la Dirección de la Escuela no presta la adecuada atención a las necesidades y requerimientos de los estudiantes de las carreras de Administración e Ingeniería Comercial; observándose que prefieren atender menesteres de indole personal y otras ocupaciones ajenas a la misión de la institución.

En la Tabla $\mathrm{N}^{\circ} 03$, se puede concluir que los estudiantes se encuentran insatisfechos con la Organización de las Carreras, puesto que el Decanato de la Facultad no presta la adecuada atención a las necesidades y requerimientos de los estudiantes de las carreras de Administración e Ingeniería Comercial: observándose que el Decano esta más concentrado en los asuntos políticos y problemas del quehacer universitario; descuidando de esta manera el acontecer diario de la Facultad.

En la Tabla $\mathrm{N}^{\circ} 04$; se observa asimismo que el curriculo, no ha resultado efectivo, porque el régimen anual para el desarrollo de las asignaturas es excesivo y el ordenamiento de las asignaturas no guarda relación con los requisitos, ya que existe una serie de deficiencias tales como: ausencia de especialización en la formación profesional, falta de una certificación progresiva que le permita al estudiante acceder a fuentes de trabajo, no se ha considerado actividades cocurriculares que permitan desarrollar capacidades de liderazgo y relaciones humanas, no se da un enfoque aplicado en la carrera, en los cursos de métodos cuantitativos y en los complementarios; $\mathrm{y}$, la falta de un enfoque más práctico y operativo a los cursos de carrera hacen que los estudiantes no se encuentren satisfechos, perjudicando

Tabla $N^{\circ} 01$ : Clases teóricas

\begin{tabular}{|c|c|c|c|c|c|c|c|c|c|c|c|c|c|}
\hline \multirow{3}{*}{$\begin{array}{l}\text { I. } \\
\mathrm{N}^{\circ}\end{array}$} & \multicolumn{13}{|c|}{ Grado de Satisfacción } \\
\hline & $\begin{array}{l}\text { Métodos de } \\
\text { Enseñanza y }\end{array}$ & \multicolumn{2}{|c|}{ Muy sat. } & \multicolumn{2}{|c|}{ Satisf. } & \multicolumn{2}{|c|}{ Algo sat. } & \multicolumn{2}{|c|}{ Insat. } & \multicolumn{2}{|c|}{ Muy insat. } & \multicolumn{2}{|c|}{ Total } \\
\hline & Clases Teóricas & Frec. & $\%$ & Frec. & $\%$ & Frec. & $\%$ & Frec. & $\%$ & Frec. & $\%$ & Frec. & $\%$ \\
\hline 1 & $\begin{array}{l}\text { Motivación del } \\
\text { docente a participar } \\
\text { en clases }\end{array}$ & 42 & 0.09 & 38 & 0.09 & 112 & 0.25 & 143 & 0.32 & 109 & 0.25 & 444 & 100 \\
\hline 2 & $\begin{array}{l}\text { Entrega de material } \\
\text { docente }\end{array}$ & 27 & 0.06 & 34 & 0.08 & 156 & 0.35 & 134 & 0.30 & 93 & 0.21 & 444 & 100 \\
\hline 3 & $\begin{array}{l}\text { Utilidad del } \\
\text { material entregado }\end{array}$ & 56 & 0.13 & 87 & 0.20 & 123 & 0.28 & 112 & 0.25 & 66 & 0.15 & 444 & 100 \\
\hline 4 & $\begin{array}{l}\text { Oportunidades de } \\
\text { realizar trabajos } \\
\text { grupales }\end{array}$ & 67 & 0.15 & 89 & 0.20 & 109 & 0.25 & 92 & 0.21 & 87 & 0.20 & 444 & 100 \\
\hline 5 & $\begin{array}{l}\text { Aplicabilidad de } \\
\text { los conocimientos } \\
\text { al campo laboral }\end{array}$ & 43 & 0.10 & 95 & 0.21 & 98 & 0.22 & 123 & 0.28 & 85 & 0.19 & 444 & 100 \\
\hline 6 & $\begin{array}{l}\text { Oportunidades de } \\
\text { discusiones durante } \\
\text { las clases }\end{array}$ & 58 & 0.13 & 98 & 0.22 & 101 & 0.23 & 99 & 0.22 & 88 & 0.20 & 444 & 100 \\
\hline 7 & $\begin{array}{l}\text { Coherencia entre lo } \\
\text { enseñado en } \\
\text { diferentes } \\
\text { asignaturas }\end{array}$ & 63 & 0.14 & 86 & 0.19 & 99 & 0.22 & 107 & 0.24 & 89 & 0.20 & 444 & 100 \\
\hline 8 & $\begin{array}{l}\text { Vocabulario y } \\
\text { material de apoyo } \\
\text { utilizado }\end{array}$ & 54 & 0.12 & 83 & 0.19 & 102 & 0.23 & 119 & 0.27 & 86 & 86.00 & 444 & 100 \\
\hline
\end{tabular}




\begin{tabular}{|c|c|c|c|c|c|c|c|c|c|c|c|c|c|}
\hline \multirow{3}{*}{$\begin{array}{l}\text { III. } \\
\text { No }\end{array}$} & \multirow{3}{*}{$\begin{array}{c}\text { Organización de } \\
\text { las Carreras } \\
\text { Dirección de la } \\
\text { Escuela } \\
\end{array}$} & \multicolumn{10}{|c|}{ Grado de Satisfacción } & & \\
\hline & & \multicolumn{2}{|c|}{ Muy sat. } & \multicolumn{2}{|c|}{ Satisf. } & \multicolumn{2}{|c|}{ Algo sat. } & \multicolumn{2}{|c|}{ Insat. } & \multicolumn{2}{|c|}{ Muy insat. } & \multicolumn{2}{|c|}{ Total } \\
\hline & & Frec. & $\%$ & Frec & $\%$ & Frec. & $\%$ & Frec & $\%$ & Frec. & $\%$ & Frec. & $\%$ \\
\hline 1 & $\begin{array}{l}\text { Calidad de la } \\
\text { atención en } \\
\text { Secretaria de cada } \\
\text { Escuela }\end{array}$ & 45 & 0.10 & 67 & 0.15 & 102 & 0.23 & 121 & 0.27 & 109 & 0.25 & 444 & 100 \\
\hline 2 & $\begin{array}{l}\text { Calidad de atención } \\
\text { del respectivo } \\
\text { Director } y / o \\
\text { coordinador }\end{array}$ & 51 & 0.11 & 45 & 0.10 & 116 & 0.26 & 134 & 0.30 & 98 & 0.22 & 444 & 100 \\
\hline 3 & $\begin{array}{l}\text { Disponibilidad de } \\
\text { tiempo para atender } \\
\text { a los estudiantes }\end{array}$ & 54 & 0.12 & 67 & 0.15 & 114 & 0.26 & 112 & 0.25 & 97 & 0.22 & 444 & 100 \\
\hline 4 & $\begin{array}{l}\text { Grado de coherencia } \\
\text { con centro de } \\
\text { alumnos }\end{array}$ & 67 & 0.15 & 74 & 0.17 & 109 & 0.25 & 112 & 0.25 & 82 & 0.18 & 444 & 100 \\
\hline
\end{tabular}

Tabla $N^{\circ} 03$ : Decanato de la Facultad

\begin{tabular}{|c|c|c|c|c|c|c|c|c|c|c|c|c|c|}
\hline \multirow{3}{*}{$\begin{array}{l}\text { III. } \\
\mathrm{N}^{\circ}\end{array}$} & \multirow{3}{*}{$\begin{array}{c}\text { Organización de las } \\
\text { Carreras } \\
\text { Decanato de la Facultad }\end{array}$} & \multicolumn{10}{|c|}{ Grado de Satisfacción } & \multirow{2}{*}{\multicolumn{2}{|c|}{ Total }} \\
\hline & & \multicolumn{2}{|c|}{ Muy sat. } & \multicolumn{2}{|c|}{ Satisf. } & \multicolumn{2}{|c|}{ Algo sat. } & \multicolumn{2}{|c|}{ Insat. } & \multicolumn{2}{|c|}{ Muy insat. } & & \\
\hline & & Frec. & $\%$ & Frec. & $\%$ & Frec. & $\%$ & Frec. & $\%$ & Frec. & $\%$ & Frec. & $\%$ \\
\hline 1 & $\begin{array}{l}\text { Calidad de la atención en } \\
\text { Secretaria }\end{array}$ & 32 & 0.07 & 43 & 0.10 & 102 & 0.23 & 152 & 0.34 & 115 & 0.26 & 444 & 100 \\
\hline 2 & $\begin{array}{l}\text { Calidad de atención del } \\
\text { Decano }\end{array}$ & 21 & 0.05 & 38 & 0.09 & 98 & 0.22 & 149 & 0.34 & 138 & 0.31 & 444 & 100 \\
\hline 3 & $\begin{array}{l}\text { Grado de compromiso con } \\
\text { los estudiantes }\end{array}$ & 38 & 0.09 & 35 & 0.08 & 111 & 0.25 & 142 & 0.32 & 118 & 0.27 & 444 & 100 \\
\hline
\end{tabular}

Tabla No 04: Malla Curricular

\begin{tabular}{|c|c|c|c|c|c|c|c|c|c|c|c|c|c|}
\hline \multirow{3}{*}{$\begin{array}{l}\text { III. } \\
\mathrm{N}^{\circ}\end{array}$} & \multirow{3}{*}{$\begin{array}{l}\text { Organización de } \\
\text { las Carreras } \\
\text { Malla Curricular }\end{array}$} & \multicolumn{10}{|c|}{ Grado de Satisfacción } & \multirow{2}{*}{\multicolumn{2}{|c|}{ Total }} \\
\hline & & \multicolumn{2}{|c|}{$\begin{array}{c}\text { Muy } \\
\text { satisfecho }\end{array}$} & \multicolumn{2}{|c|}{ Satisfecho } & \multicolumn{2}{|c|}{$\begin{array}{c}\text { Algo } \\
\text { satisfecho }\end{array}$} & \multicolumn{2}{|c|}{ Insatisfecho } & \multicolumn{2}{|c|}{$\begin{array}{c}\text { Muy } \\
\text { insatisfecho }\end{array}$} & & \\
\hline & & Frec. & $\%$ & Frec. & $\%$ & Frec. & $\%$ & Frec. & $\%$ & Frec. & $\%$ & Frec. & $\%$ \\
\hline 1 & $\begin{array}{l}\text { Estructura de la } \\
\text { Malla Curricular }\end{array}$ & 27 & 0.06 & 64 & 0.14 & 102 & 0.23 & 142 & 0.32 & 109 & 0.25 & 444 & 100 \\
\hline 2 & $\begin{array}{l}\text { Congruencia de } \\
\text { las asignaturas } \\
\text { cubiertas por la } \\
\text { Malla }\end{array}$ & 23 & 0.05 & 76 & 0.17 & 116 & 0.26 & 134 & 0.30 & 95 & 0.21 & 444 & 100 \\
\hline 3 & $\begin{array}{l}\text { Cantidad de } \\
\text { cursos por } \\
\text { semestre y/o año } \\
\text { académico }\end{array}$ & 27 & 0.06 & 75 & 0.17 & 128 & 0.29 & 122 & 0.27 & 92 & 0.21 & 444 & 100 \\
\hline
\end{tabular}




\begin{tabular}{|c|c|c|c|c|c|c|c|c|c|c|c|c|c|}
\hline \multirow{3}{*}{$\begin{array}{l}\text { IV. } \\
\mathrm{N}^{\circ}\end{array}$} & \multirow{3}{*}{$\begin{array}{c}\text { Personal Docente } \\
\text { Docentes }\end{array}$} & \multirow{3}{*}{$\begin{array}{l}\text { Muy } \\
\text { sat. } \\
\text { Frec. }\end{array}$} & \multicolumn{8}{|c|}{ Grado de Satisfacción } & \multirow{2}{*}{\multicolumn{3}{|c|}{ Total }} \\
\hline & & & \multicolumn{2}{|c|}{ Satisf. } & \multicolumn{2}{|c|}{ Algo sat. } & \multicolumn{2}{|c|}{ Insat. } & \multicolumn{2}{|c|}{ Muy insat. } & & & \\
\hline & & & $\%$ & Frec. & $\%$ & Frec. & $\%$ & Frec. & $\%$ & Frec. & $\%$ & Frec. & $\%$ \\
\hline 1 & $\begin{array}{l}\text { Disponibilidad de } \\
\text { atención en oficina y/o } \\
\text { departamento }\end{array}$ & 44 & 0.10 & 53 & 0.12 & 108 & 0.24 & 143 & 0.32 & 96 & 0.22 & 444 & 100 \\
\hline 2 & Trato con los estudiantes & 42 & 0.09 & 54 & 0.12 & 121 & 0.27 & 134 & 0.30 & 93 & 0.21 & 444 & 100 \\
\hline 3 & $\begin{array}{l}\text { Calidad académica de los } \\
\text { docentes }\end{array}$ & 45 & 0.10 & 62 & 0.14 & 93 & 0.21 & 143 & 0.32 & 101 & 0.23 & 444 & 100 \\
\hline 4 & $\begin{array}{l}\text { Puntualidad y } \\
\text { cumplimiento de horas de } \\
\text { clases }\end{array}$ & 44 & 0.10 & 48 & 0.11 & 99 & 0.22 & 132 & 0.30 & 121 & 0.27 & 444 & 100 \\
\hline 5 & $\begin{array}{l}\text { Claridad en la exposición } \\
\text { de contenidos }\end{array}$ & 46 & 0.10 & 61 & 0.14 & 96 & 0.22 & 119 & 0.27 & 122 & 0.27 & 444 & 100 \\
\hline 6 & $\begin{array}{l}\text { Motivación a los } \\
\text { estudiantes a participar en } \\
\text { clases }\end{array}$ & 49 & 0.11 & 78 & 0.18 & 96 & 0.22 & 109 & 0.25 & 112 & 0.25 & 444 & 100 \\
\hline 7 & $\begin{array}{l}\text { Cumplimiento con el } \\
\text { programa del curso }\end{array}$ & 41 & 0.09 & 66 & 0.15 & 92 & 0.21 & 139 & 0.31 & 106 & 0.24 & 444 & 100 \\
\hline 8 & $\begin{array}{l}\text { Disposición de los } \\
\text { docentes en la atención de } \\
\text { las consultas }\end{array}$ & 48 & 0.11 & 74 & 0.17 & 91 & 0.20 & 112 & 0.25 & 119 & 0.27 & 444 & 100 \\
\hline
\end{tabular}

de esta manera su nivel de formación académico profesional.

Finalmente en la tabla $\mathrm{N}^{\circ} 05$; se muestran resultados que nos permiten inferir que los estudiantes de la Facultad de Administración se encuentran insatisfechos con el Personal Docente de la Facultad; puesto que su comportamiento y calidad académica resta la formación académico profesional de los estudiantes y por lo tanto no satisface las expectativas de los mismos.

\section{CONCLUSIONES}

1. El nivel de la calidad del sistema de formación profesional que se brinda en la Facultad de Ciencias Administrativas de la UNJBG de Tacna, es bajo; puesto que el Nivel de enseñanza y aprendizaje, el tipo de evaluaciones, el nivel del equipo docente, el nivel de organización de las escuelas profesionales, el nivel de Infraestructura; $y$, el nivel de la Curricula de estudios no satisfacen las expectativas académico profesionales de los estudiantes.

2. Los estudiantes de la Facultad de Ciencias Administrativas de la UNJBG de Tacna se encuentran insatisfechos respecto a la calidad del sistema de formación profesional; puesto que los elementos que la conforman no se encuentran acordes con la formación profesional en el campo de la Administración y la Empresa.

3. La calidad del sistema de formación profesional incide en el nivel de satisfacción de los estudiantes de la Facultad de Ciencias Administrativas de la UNJBG de Tacna, período 2009; habiéndose encontrado que existen indicadores que no cumplen con la satisfacción de necesidades y requerimientos de los estudiantes y futuros profesionales de las carreras de Administración e Ingenieria Comercial.

\section{REFERENCIAS BIBLIOGRÁFICAS}

Arrien. Juan. Calidad y Acreditación exigencias a la universidad.

Bernal, J.B. (2003) La Calidad: desafío que enfrenta la educación en el momento actual. Proyecto UNESCO/Países Bajos. (SIMED), San José.

Brennan, John. (2005) "Panorama General del Aseguramiento de Calidad". México.

Brünner, José Joaquín (1991). "La reforma de 1980. Diezaños después". Mimeo.

Brünner J.J. (2005). "Educación superior, integración económica y globalización". En: Primer Simposio Regional "Educación, Trabajo y la Integración Económica del Merconorte". Consejo de Educación Superior de Puerto Rico, San Juan. (26 de septiembre).

Brünner, José Joaquin (1997). "Calidad y Evaluación en la Educación Superior" en: "evaluación y Acreditación Universitaria". Metodologias y experiencias". Eds. Mario Letelier y Eduardo Martinez. Caracas. Venezuela, Nueva Sociedad, UNESCO.

Cabero, J. (2000). Análisis de Medios de Enseñanza. Edic. Alfar., Sevilla.

Calderón Ramírez, Jorge (2000). "Calidad en el Aula. Una aspiración permanente" Calidad Integral, Centro de 
Capacitación, S.C., Guadalajara, Jal. México

Cámara de Diputados. (1997). Desafíos actuales de la Educación Superior. Mimeo.

Daghlian, Jacob, (2004) Educación para la Calidad y Calidad en la Educación.

De la Orden Hoz, Arturo. (2003). "Desarrollo y Validación de un Modelo de Calidad Universitaria como base para su Evaluación". Revista Electrónica de Investigación y Evaluación Educativa. Volumen 3. Número 1-2.

Fernández Diaz. Ma José y González Galán Arturo. (2001). "Desarrollo y Situación Actual de los Estudios de Eficacia Escolar". Revista Electrónica de Investigación y Evaluación Educativa. Volumen 3. Número 13.

GIMENO, J. (2002): "Reformas educativas. Utopia, retórica y práctica", Cuadernos de Pedagogia, ${ }^{\circ} 209$. pp. 62-68.
Gonzales, Juan (2000), Bases para la Construcción de Indicadores de Gestión Universitaria, Universidad Católica de Santa María.

Herrera, Ricardo. (1998). "Bases para el desarrollo cualitativo de la docencia universitaria". Gestión de la Docencia e Internalización en universidades chilenas. CINDA.

\section{Correspondencia:}

Silvia Munarriz O'phelan

Ciudad Universitaria - Fundo "Los Granados"

Av. Miraflores s/n. Tacna. Perú

silvia mo_25@hotmail.com 